SANDIA REPORT

SAND2009-6194

Unlimited Release

Printed September 2009

\title{
Feasibility of Neuro-Morphic Computing to Emulate Error-Conflict Based Decision Making
}

Darren W. Branch, Conrad D. James, Chris Forsythe

Prepared by

Sandia National Laboratories

Albuquerque, New Mexico 87185 and Livermore, California 94550

Sandia is a multiprogram laboratory operated by Sandia Corporation, a Lockheed Martin Company, for the United States Department of Energy's National Nuclear Security Administration under Contract DE-AC04-94AL85000.

Approved for public release; further dissemination unlimited. 
Issued by Sandia National Laboratories, operated for the United States Department of Energy by Sandia Corporation.

NOTICE: This report was prepared as an account of work sponsored by an agency of the United States Government. Neither the United States Government, nor any agency thereof, nor any of their employees, nor any of their contractors, subcontractors, or their employees, make any warranty, express or implied, or assume any legal liability or responsibility for the accuracy, completeness, or usefulness of any information, apparatus, product, or process disclosed, or represent that its use would not infringe privately owned rights. Reference herein to any specific commercial product, process, or service by trade name, trademark, manufacturer, or otherwise, does not necessarily constitute or imply its endorsement, recommendation, or favoring by the United States Government, any agency thereof, or any of their contractors or subcontractors. The views and opinions expressed herein do not necessarily state or reflect those of the United States Government, any agency thereof, or any of their contractors.

Printed in the United States of America. This report has been reproduced directly from the best available copy.

Available to DOE and DOE contractors from

U.S. Department of Energy

Office of Scientific and Technical Information

P.O. Box 62

Oak Ridge, TN 37831

Telephone: $\quad$ (865)576-8401

Facsimile: $\quad$ (865)576-5728

E-Mail: reports@adonis.osti.gov

Online ordering: http://www.osti.gov/bridge

Available to the public from

U.S. Department of Commerce

National Technical Information Service

5285 Port Royal Rd

Springfield, VA 22161

Telephone: $\quad$ (800)553-6847

Facsimile: $\quad$ (703)605-6900

E-Mail: orders@ntis.fedworld.gov

Online order: http://www.ntis.gov/help/ordermethods.asp?loc=7-4-0\#online

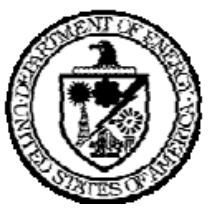


SAND2009-6194

Unlimited Release

Printed September 2009

\title{
Feasibility of Neuro-Morphic Computing to Emulate Error-Conflict Based Decision Making
}

\author{
Darren W. Branch \\ Biosensors and Nanomaterials Department \\ Sandia National Laboratories \\ PO Box 5800 \\ Albuquerque, NM 87185-1425
}

\begin{abstract}
A key aspect of decision making is determining when errors or conflicts exist in information and knowing whether to continue or terminate an action. Understanding the error-conflict processing is crucial in order to emulate higher brain functions in hardware and software systems. Specific brain regions, most notably the anterior cingulate cortex (ACC) are known to respond to the presence of conflicts in information by assigning a value to an action. Essentially, this conflict signal triggers strategic adjustments in cognitive control, which serve to prevent further conflict. The most probable mechanism is the ACC reports and discriminates different types of feedback, both positive and negative, that relate to different adaptations. Unique cells called spindle neurons that are primarily found in the ACC (layer $\mathrm{Vb}$ ) are known to be responsible for cognitive dissonance (disambiguation between alternatives). Thus, the ACC through a specific set of cells likely plays a central role in the ability of humans to make difficult decisions and solve challenging problems in the midst of conflicting information. In addition to dealing with cognitive dissonance, decision making in high consequence scenarios also relies on the integration of multiple sets of information (sensory, reward, emotion, etc.). Thus, a second area of interest for this proposal lies in the corticostriatal networks that serve as an integration region for multiple cognitive inputs. In order to engineer neurological decision making processes in silicon devices, we will determine the key cells, inputs, and outputs of conflict/error detection in the ACC region. The second goal is understand in vitro models of corticostriatal networks and the impact of physical deficits on decision making, specifically in stressful scenarios with conflicting streams of data from multiple inputs. We will elucidate the mechanisms of cognitive data integration in order to implement a future corticostriatal-like network in silicon devices for improved decision processing.
\end{abstract}




\section{TABLE OF CONTENTS}

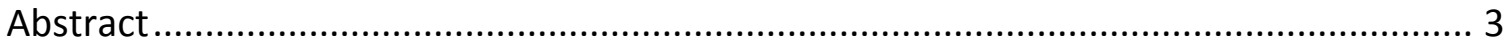

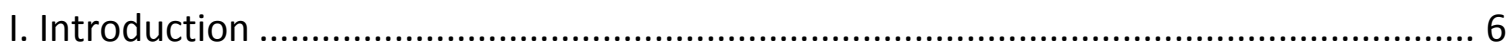

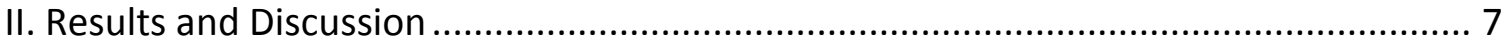

A. Computational Models of ACC-PFC ...................................................................... 7

I. Choice Discrimination Model (Conflict Type Model).......................................... 7

II. Extended Time-Scale Model............................................................................... 9

III. Error-Likelihood Model..................................................................................... 10

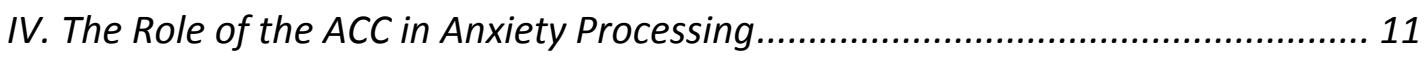

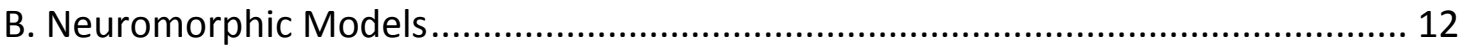

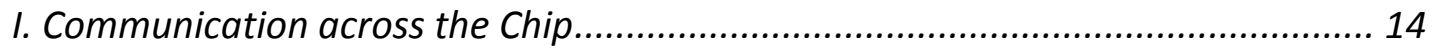

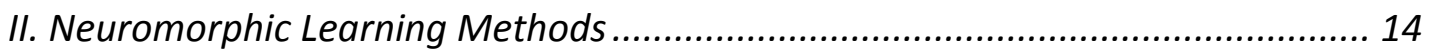

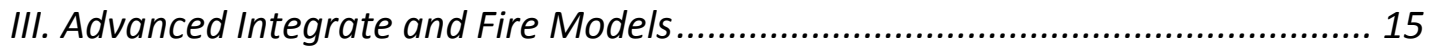

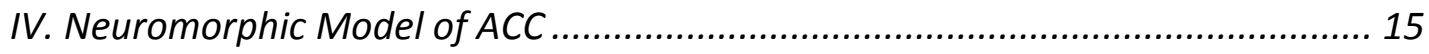

C. Patterning, Topographical, and Organizational Methods for Neural Cell Cultures.. 16

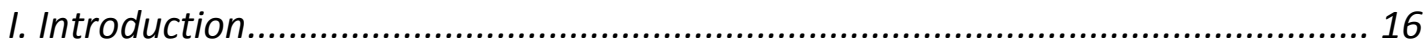

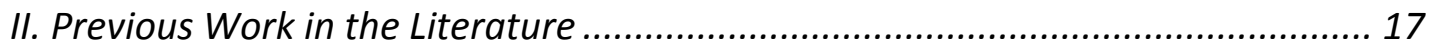

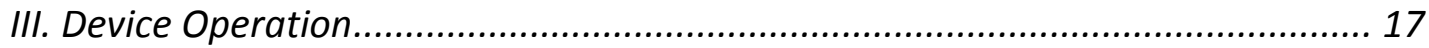

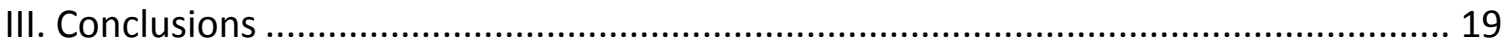

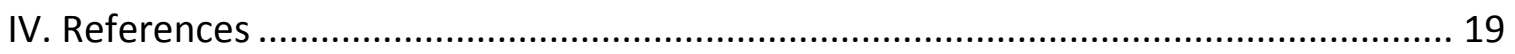

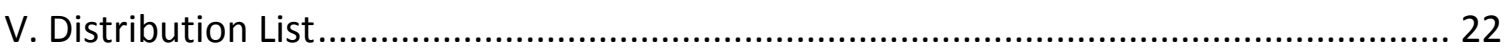




\section{Introduction}

According to the conflict-monitoring model, information processing conflict registered in the anterior cingulate cortex (ACC) triggers the prefrontal cortex to reduce conflict susceptibility. This conflict monitoring model suggests that control is modulated through a system situated in the ACC, which constantly extracts from ongoing processing information an abstract index of information conflict [1]. It has been determined that a higher value of this index triggers regulative control sites in the prefrontal cortex to boost activity. The goal of this study is to determine which prevailing ACC model is the most relevant for implementation into a solid-state format such as a neuromorphic computational process.

Neuroimaging studies show that the anterior cingulate cortex (ACC) is critically involved in cognitive control [2]. Moreover, neuroimaging literature has demonstrated that the ACC is activated during a wide range of tasks that involve response conflict, such as the Stroop [3], the Eriksen [4] and the go/no-go [5] tasks. Of the models investigated in this study, most were based on idea that the ACC detects errors as discrepancies between actual and intended events [6]. We refer to these models as the conventional ACC conflict-control loop model. Other models proposed that the ACC may detect conflict between mutually incompatible response processes such as incorrect versus correct responses [2]. Though the models examined account for most of the ACC behavior, it remains unclear how the brain learns to exert cognitive control over behavior to resolve conflicts. A more recent computational model suggests that the ACC assigns a prediction of error likelihood [7]. This model specifically predicts that the ACC response to a given task will be proportional to the perceived likelihood of an error.

A well cited example of conflict-monitoring at work is whether the current trial $n$ is impacted by the result of trial $n-1$. Based on this line of thought, any conflict on trial $n-1$ should tighten the controls on trial $n$. The type of trial appropriate from cognitive control is the Flanker test, which measures congruency (i.e. attention span) over a set of trials. The idea of the $n-1$ trials leading to tighter controls on the subsequent trials seems to be invalid according to a recent study [8]. The primary finding was reduced activation on incongruent trials than on congruent trials, suggesting that the ACC does not self regulate but merely registers the conflict.

The ACC is also known to communicate with the lateral region of prefrontal cortex (PFC) which has been studies extensively using neuroimaging [2] and neural network models [1]. Conflict can be thought of as the simultaneous coactivation of incompatible responses, such as trying to name the ink color of the word red written in green ink as in the Stroop test. In some cases conflicting tasks generate inappropriate responses, which require cognitive control to mediate the source of the conflict. The early models used to assess conflict monitoring were based on a structured conflict-control loop in Figure 1, where the ACC strictly serves as a conflict monitor. This view is in debate as will be discussed in the following sections. 


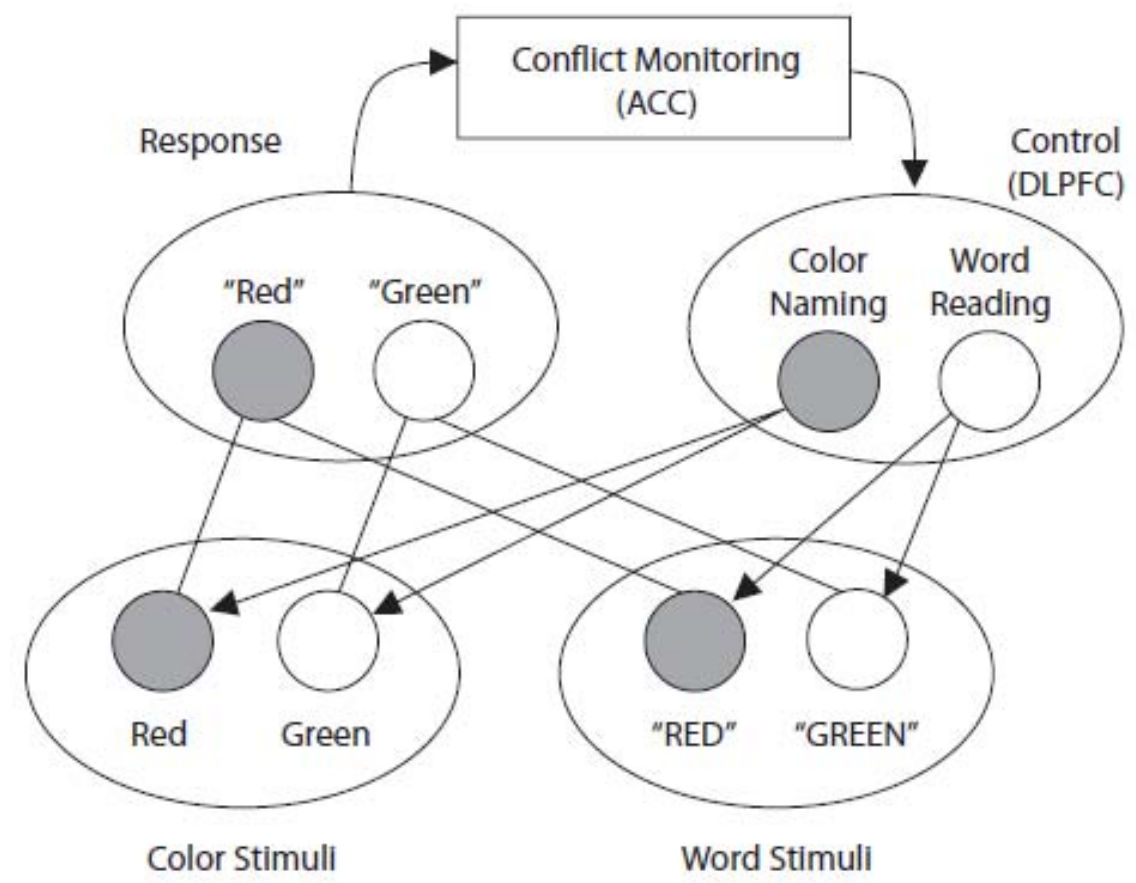

Figure 1. Example of a computational model of an interference task. When relevant (color) and irrelevant (word) stimulus dimensions are incongruent and activate conflicting responses, the anterior cingulate cortex ( $\mathrm{ACC}$ ) detects this conflict and engages attentional control mechanisms in the dorsolateral prefrontal cortex (DLPFC).

Figure 1 Conventional conflict-control model. [9]

The commonly accepted conflict control theory of the ACC was originally based on data obtained from speeded response tasks and has received reasonable validation from $\mathrm{fMRI}$ and lesion studies. Of significance is that when the ACC is lesioned, animals have difficulty using preceding trial history and fail to work as hard for rewards. This suggests that the conflict monitor, namely the ACC is unable to mediate the task conflict. Thus, the neural region cognitive control is thought to comprise an evaluative component located in the anterior cingulate cortex (ACC) and an executive component in the prefrontal cortex (PFC). The control mechanism itself is thought to be mainly local, triggered by response conflict (monitored by the ACC) and involved in the allocation of executive resources (recruited by the PFC) in a trial-to-trial fashion. In the following discussion we review three widely cited models and discuss their implementation in a neuromorphic format.

\section{Results and Discussion}

\section{A. Computational Models of ACC-PFC}

\section{Choice Discrimination Model (Conflict Type Model)}

Reference: [10]

In this model the three distinct speeded response tasks (two-alternative forced choice, go/no-go, and oddball) were examined. The idea of these tasks was to isolate the 
common factor of stimulus frequency, which modulates the degree of conflict present on any given trial. Stimulus frequency could alter the global response and/or the local sequential history or both. The neural network model in Figure 2 exhibited the following characteristics:

1) More difficult to respond appropriately to low-frequency events.

2) For task performance, the conflict index from the model closely resembled the frequency-related pattern of ACC activation.

3) The sequential history task study revealed that the model demonstrated that performance on two-response tasks can affected by subtle differences in sequential history.

4) The local sequential history rather than global frequency modulates the ACC activity in both one and two response tasks.

5) Direct detection of conflict leads to control adjustments.

6) Premature activation did not generate incorrect responses since the network can recover from an error under the one task situation.

\title{
Model Architecture
}

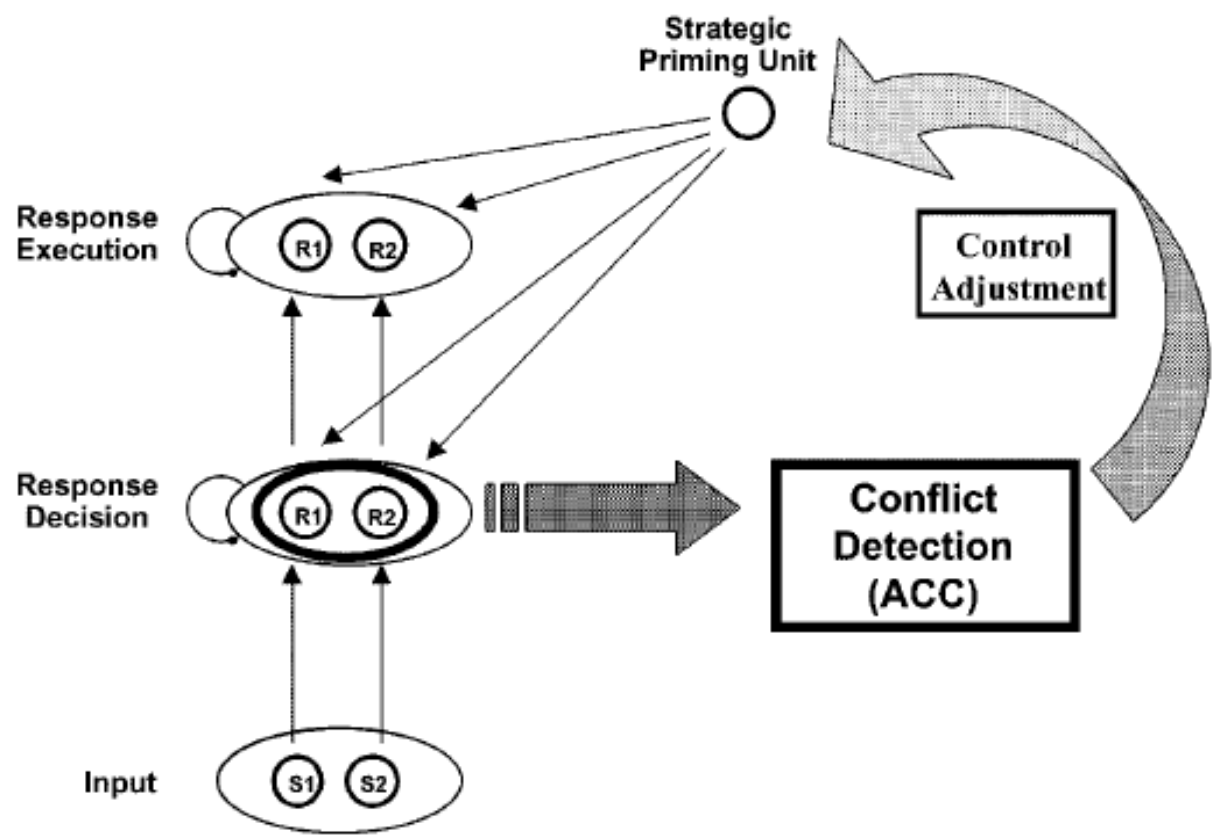

\begin{abstract}
Figure 2. Schematic diagram of model architecture. The network consisted of three feedforward layers with lateral inhibitory connections at the response decision and response execution layers. Conflict was measured at the decision layer, and modulated activity in the decision and execution layers via a strategic priming unit. Repetition priming and alternation priming (not shown) were also applied to the decision and execution layers.
\end{abstract}

Figure 2 Neural network model of the ACC implemented by [10].

This model extended the conventional ACC conflict-control model. This was accomplished by using three different tasks (e.g. go/no go, oddball, two-alternative forced choice) to tap a common factor, namely the stimulus frequency that modulates 
the degree of conflict present in a given trial. Neuroimaging data shows significantly increased ACC activity for low-frequency stimuli, relative to high-frequency stimuli [11]. This model accurately predicted this behavior in the task performance trials by closely resembling the frequency-related patterns of ACC activation [10].

\section{Extended Time-Scale Model}

Reference: [12]

The interaction of the PFC and ACC regions has largely been performed using neural network models in terms of a single conflict-control loop mechanism [2]. In the case of a single control-loop the performance of a certain task leads to detection of a response conflict, which then leads to an increase in cognitive control, resulting in conflict resolution. One neural network model in particular made significant improvements by adding an additional control loop to model the interaction between the ACC and PFC [12]. A key aspect of this model was the addition of sustained active maintenance of task-set information contained in a separate model of the PFC which was driven by a long-time scale conflict on the order of several minutes. The goal of this new model was to resolve discrepancies between empirical data on human behavior and brain activation. The task for this neural network model is the color naming Stroop test. Briefly, the task requires verbally responding with the name of the font color when presented as a word. Examples include "GREEN" written in green designate a congruent trial, DOG written green is neutral and "RED" written in blue is incongruent. In Figure 3 a neural network model was constructed to capture the physiological pathways of the ACC-PFC regions. In the model connectivity strength between the "word" and "response" were increased over the connection with the "color" layer to capture the asymmetrical behavior.

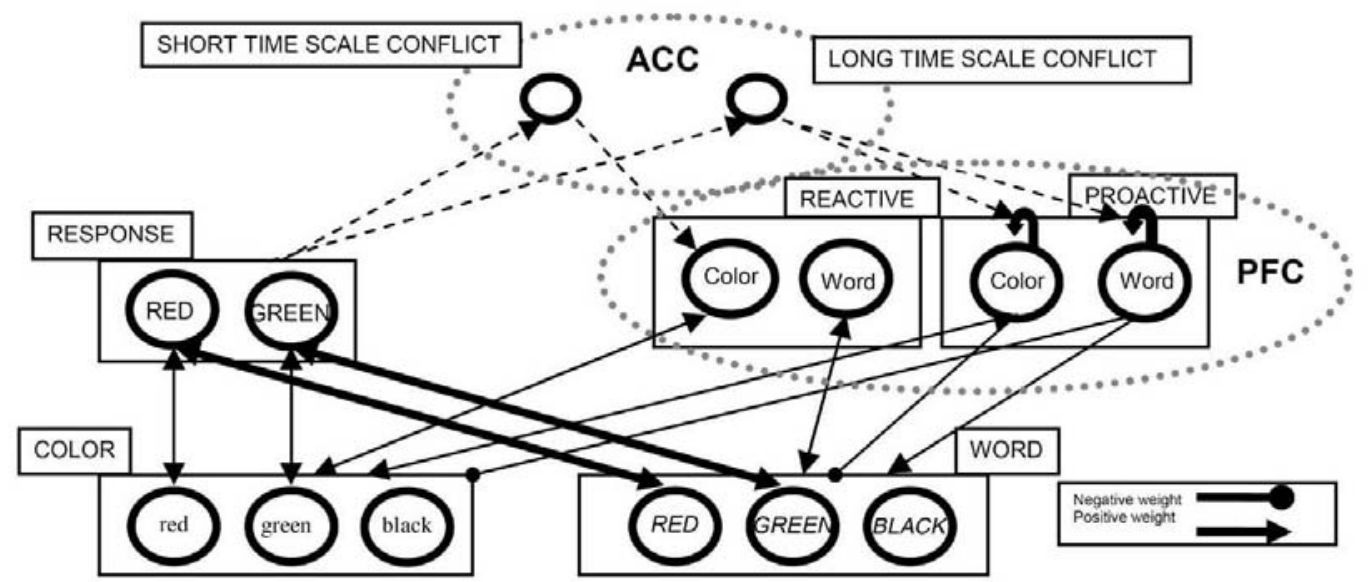

Fig. 1. A model of dual mechanisms in cognitive control in the color-naming Stroop test. Excitatory connections (arrowheads) impinging on units represent unit-specific inputs; connections impinging on network layers (represented by rectangles) represent inputs to the entire layer. Inhibition (circleheads) is also present within each layer. For detailed description and equations see main text and Table 1.

Figure 3 Large scale connectionist network of interaction between ACC and PFC. [12]. This model is an extended form of the one developed by [1].

This model had the following features: learning was absent, lateral inhibition within each layer ensured competitive dynamics, and noise was present. The Hopfield energy 
was calculated for conflict across a short time-scale with a second conflict set for a long time-scale. The long time-scale conflict parameter remained constant for each trial and was computed as an average of previous short time-scale conflicts. This long time-scale conflict input had the tendency to exert more control following high levels of experienced conflict. A key issue of debate is whether the level of experience impacts subsequent trials and therefore models with this behavior may not be physically relevant under certain cases. For analysis results from trials developed for this model were compared with brain imaging taken from fMRI. This model appears to capture several notable behaviors of the ACC-PFC region:

1) Local and sustained experience of conflict during performance might lead to a shift in the neural mechanisms of cognitive control engaged to perform the task.

2) Since individuals were not aware of changes in the trials, the adjustments had to occur implicitly without conscience intervention.

Yet this model lacks a learning element that is characteristic of all other models. It seems apparent that this model may only capture a small subset of the ACC behavior. Key issues with the conflict model include:

1) Fails to show how the ACC contributes to decision making.

2) There is no mechanism for context-sensitive learning which is critical for adaptive behavior.

\section{Error-Likelihood Model}

Reference: $[7,13]$

In this model the hypothesis is that the ACC assigns a prediction of error likelihood and that the response of the ACC to a given task condition is proportional to the perceived error likelihood. The goals were to examine how ACC representations might develop through experience and explicitly investigate the implications of this hypothesis. 

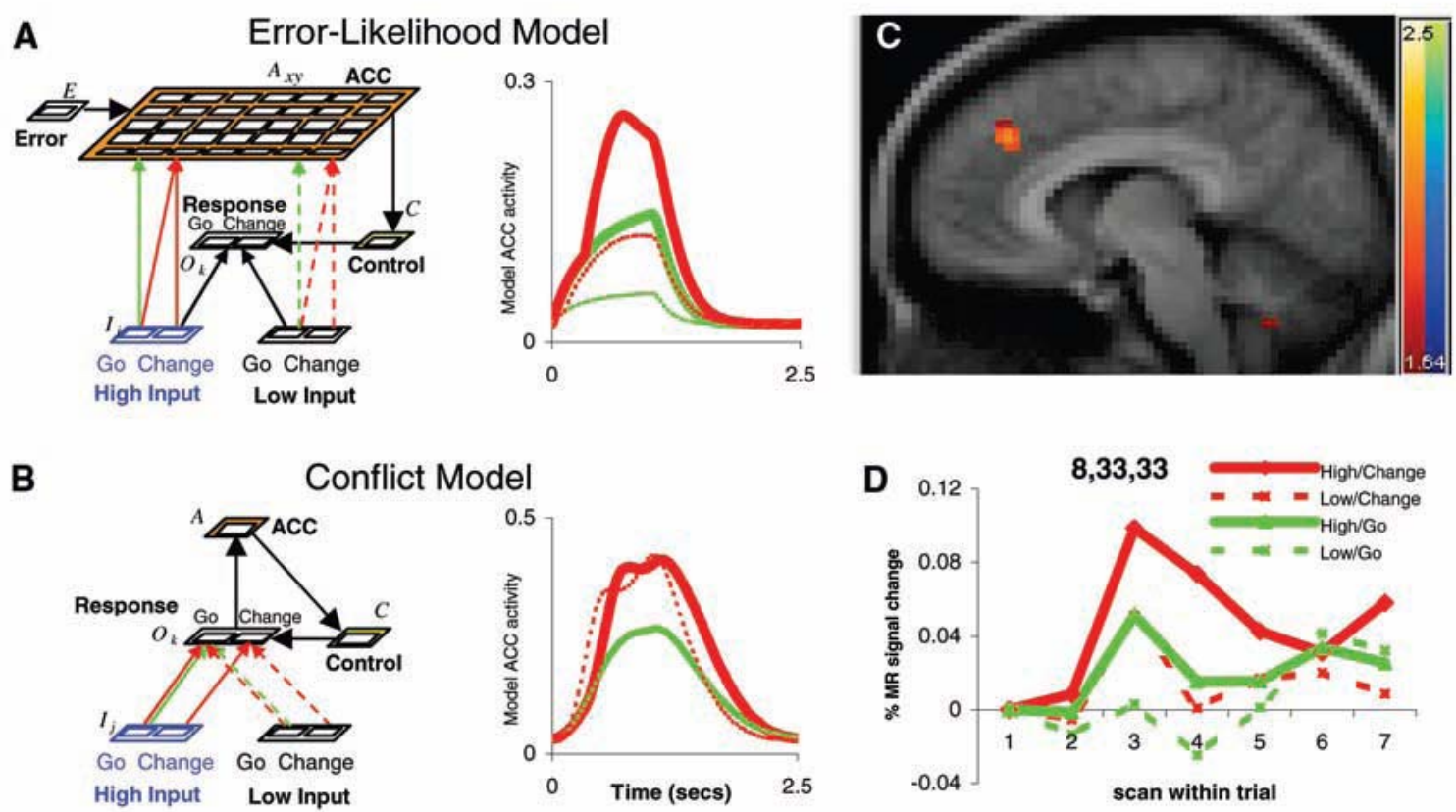

Figure 4 Error-Likelihood model versus the conflict model [7]. Only the error-likelihood model predicts effects of high-error-versus low-error-likelihood conditions in the low-conflict go (thick green versus thin green lines) as well as high conflict change conditions (thick red versus thin red lines).

This model has the following characteristics:

1. Substantiates support for error driven ACC function in which the ACC learns to signal from the magnitude of its activity, which conflict based models have not yet attempted to do.

2. The predicted likelihood of an error occurs in response to a given task condition.

3. Possibly able to recruit cognitive control to match predicted demand.

4. Consistent with the idea that a dopaminergic training signal in ACC is involved in a common role in reinforcement learning and cognitive recruitment.

5. ACC serves a general function of detecting and avoiding risk.

The error-likelihood model appears to capture a majority of the observed behavior for the ACC region and offers specific improvements over the conflict model. One major drawback is that recent experiments fail to find effects on ACC activation from cues that signal error likelihood. Yet a clear advantage of this model is the ability to account well for learning effects, a behavior that is lacking in the conflict-control loop models.

\section{The Role of the ACC in Anxiety Processing}

The ACC region of the brain plays a critical role in the processing of error and conflict information. However, more recently the ACC has been involved in affective and anxiety processing [14]. For example, individuals with anxious behavior showed altered biases when attempting to disambiguate affective information. In this case the appraisal task was to assess the emotional states of faces with specific emotional expressions. In particular, anxiety prone individuals activate the dorsal ACC more (and the ventral ACC less) during decision-making in the context of affective ambiguity, and the amount of 
activation in the dorsal ACC relates to the bias in the capacity to detect facial expressions [14]. This behavior of the ACC may be interesting to implement in a solidstate computational model given the activation levels in different parts of the ACC.

\section{B. Neuromorphic Models}

Neuromorphic circuits are solid-state implementation of the biological systems in a silicon-chip. In this case the neuron behavior is based on physiological recordings that are modeled using differential equations. In this formalism the ion-channel behavior, synapse location, distribution, spike rate, connectivity, and overall organization are represented by an analogue system implemented in silicon transistors. By operating the transistors in a sub-threshold state, power dissipation levels are now compatible with very large scale integration. Using conventional CMOS technology, scalable architectures of neural networks have been developed that operate as bidirectional associative memories (BAM) [15]. The scalability depends on the physical behavior that can be captured by a computational element (i.e. transistors). An example of implementing neurons in VLSI is shown in Figure 5. This early study was aimed at determining the computational penalty associated with using several transistors to perform neural-based computations.

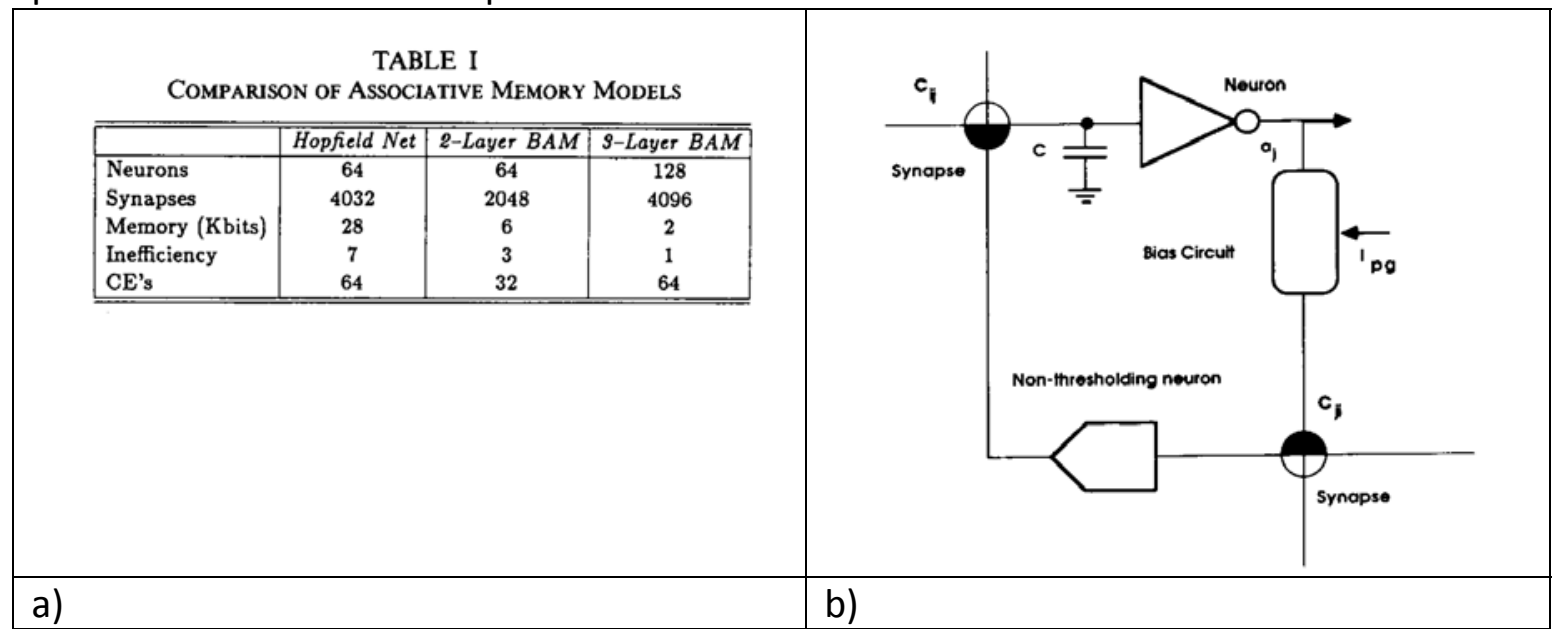

Figure 5 Implementing neurons in VLSI. a) Efficiency of Implementation in VLSI CE: computing element that can perform a multiplication or addition operation. Inefficiency is defined as hardware bits per information bits, b) Simple synthetic neural circuit. The synapses are programmable transconductances and the capacitance served as interconnects. These neurons are thresholding and nonthresholding neuron. A bias circuit allows the current levels to be externally programmed.

These elements in Figure $5 \mathrm{~b}$ can be combined to implement the three layer architecture of a BAM [15] as shown in Figure 6. 


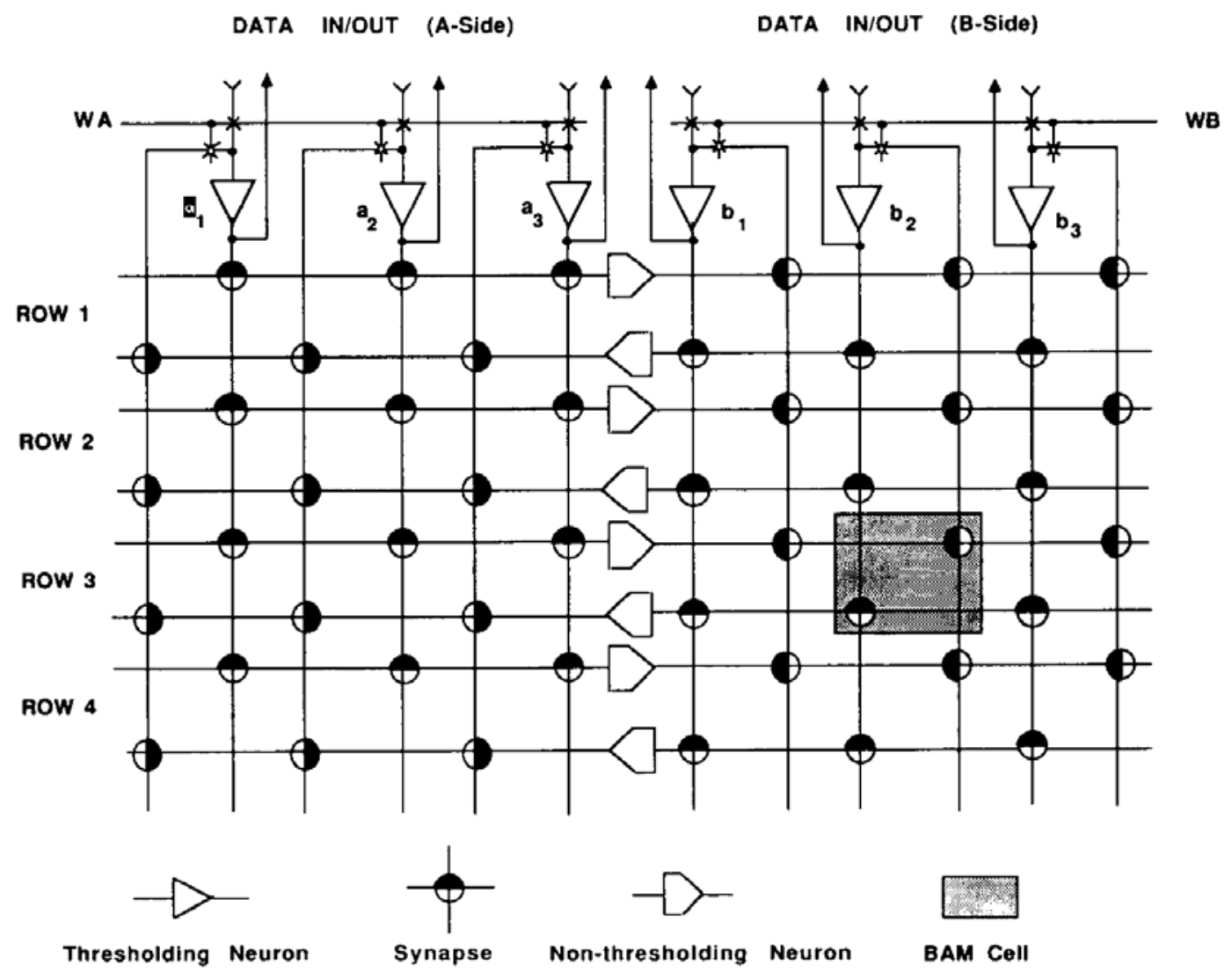

Figure 6 Three layer BAM circuit for neural chip design.

Using these constructs for neurons, cortical pyramidal cell have been modeled in silicon. The circuit consisted of an n-type current carrier and a p-type winner-takes-all (WTA) circuit. The WTA architecture selects one node out of many through competition mechanisms that depend on the amplitude of the architecture input signals. These circuits are useful optimizing power consumption. In this case, the WTA circuit current conveyors compete for current supplied to a common line. When a WTA cell and current conveyor feed each other, their current buffering devices, M1 and M2 act as common source amplifiers. The result is an unstable positive feedback loop and $X_{v}$ and $X_{L}$ go to the voltage rails. The additional transistor prevents the device from entering the linear range by driving node $X_{v}$ to maintain saturation of the buffer current (Figure 7). 


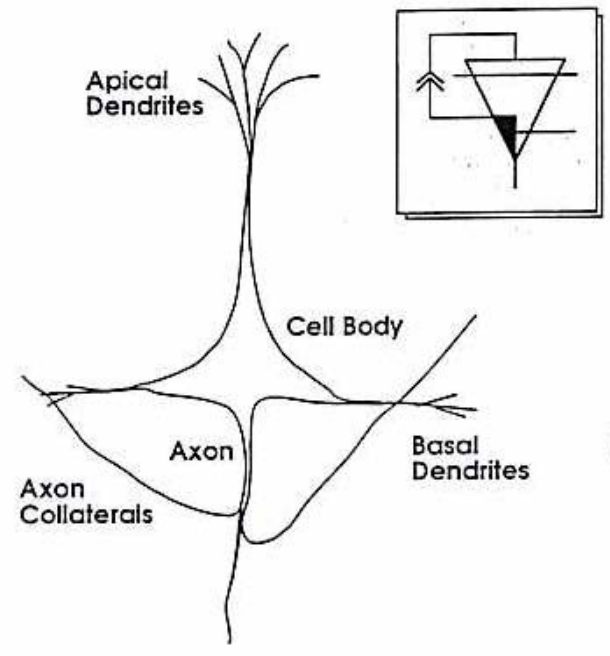

(a)

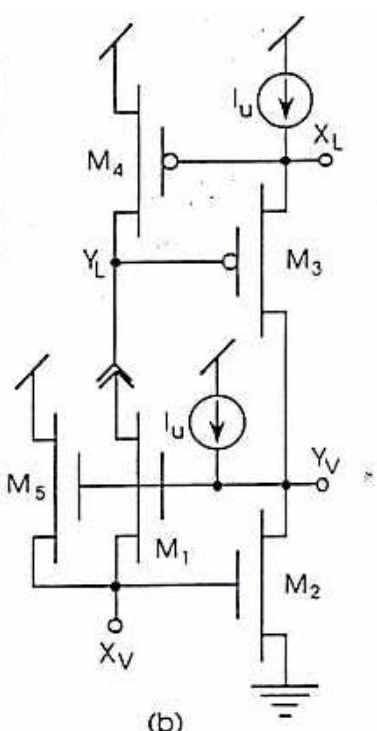

(b)

Fig. 17.10. (a) Cortical pyramidal cell. Inputs are received at the apical and basal dendrites; the cell's output is communicated by its axon. (b) Pyramidal cell circuit. An n-type current conveyor communicates inputs and outputs through node $\mathrm{X}_{V}$ and a p-type WTA cell realizes lateral inhibition through node $\mathrm{X}_{L}$. The circuit symbol includes the current conveyor's control and supply nodes and the WTA's input, as well as the communication nodes.

Figure 7 Circuit for cortical pyramidal cell [16].

Rather than implementing abstract neural networks that may only partially resemble a physical process, neuromorphic systems are hardware devices, containing analog circuits that attempt to model physical processes at the device physics level. In the past several years dedicated neuromorphic circuits have been constructed for various biological processes. Recent neuromorphic studies range from cochlear implantable processors [17], massive parallel networks of integrate and fire neurons [18], speech recognizers, sonar chips based on bat echolocation, and silicon retinas [19].

\section{Communication across the Chip}

The strategy used by neuromorphic devices to communicate analog signals across chip boundaries uses an asynchronous communication protocol. Analog signals are converted into streams of non-clocked digital pulses (spikes) and encoded using pulsefrequency modulation (spike rates). This method is called address-event representation (AER). In AER, each analog element is assigned an address. When a spiking element generates a pulse its address is instantaneously put on a digital bus using asynchronous logic. Address events are the digital pulses written to the bus. Systems containing multiple AER chips can be constructed by implementing special purpose off-chip arbitration schemes

\section{Neuromorphic Learning Methods}

While mean rate Hebbian learning algorithms are difficult to implement using analog circuits, spike-timing-based learning rules map directly onto silicon [20]. A promising class of spike-driven learning rules that is particularly well suited to VLSI implementation is the one based on the spike-timing dependent plasticity (STDP) mechanism [21]. In STDP the precise timing of spikes generated by the pre and postsynaptic neurons shape 
the synaptic efficacy. If a presynaptic spike arrives at the synaptic terminal before a postsynaptic spike is emitted, within a critical time window, the synaptic efficacy is increased. Conversely, if the postsynaptic spike is emitted before the presynaptic one arrives, the synaptic efficacy is decreased. Several modeling studies have developed learning algorithms based on STDP, and demonstrated how systems that use these types of algorithms can carry out complex information processing tasks [22].

\section{Advanced Integrate and Fire Models}

Generally, VLSI I\&F neurons integrate presynaptic input currents and generate a voltage pulse when the integrated voltage reaches a threshold. A very simple but influential circuit implementation of this model is the "Axon-Hillock." In this circuit, an integrating capacitor is connected to two inverters, a feedback capacitor, and a reset transistor driven by the output inverter. The Axon-Hillock circuit is very compact, comprising only six transistors and two capacitors, but it has a major drawback: it dissipates significant amounts of power. An example of an I\&F circuit optimized with respect to power consumption in shown in Figure 8. This circuit is optimized for power consumption and implements spike-frequency adaptation as well as a tunable refractory period, and voltage threshold modulation [18].

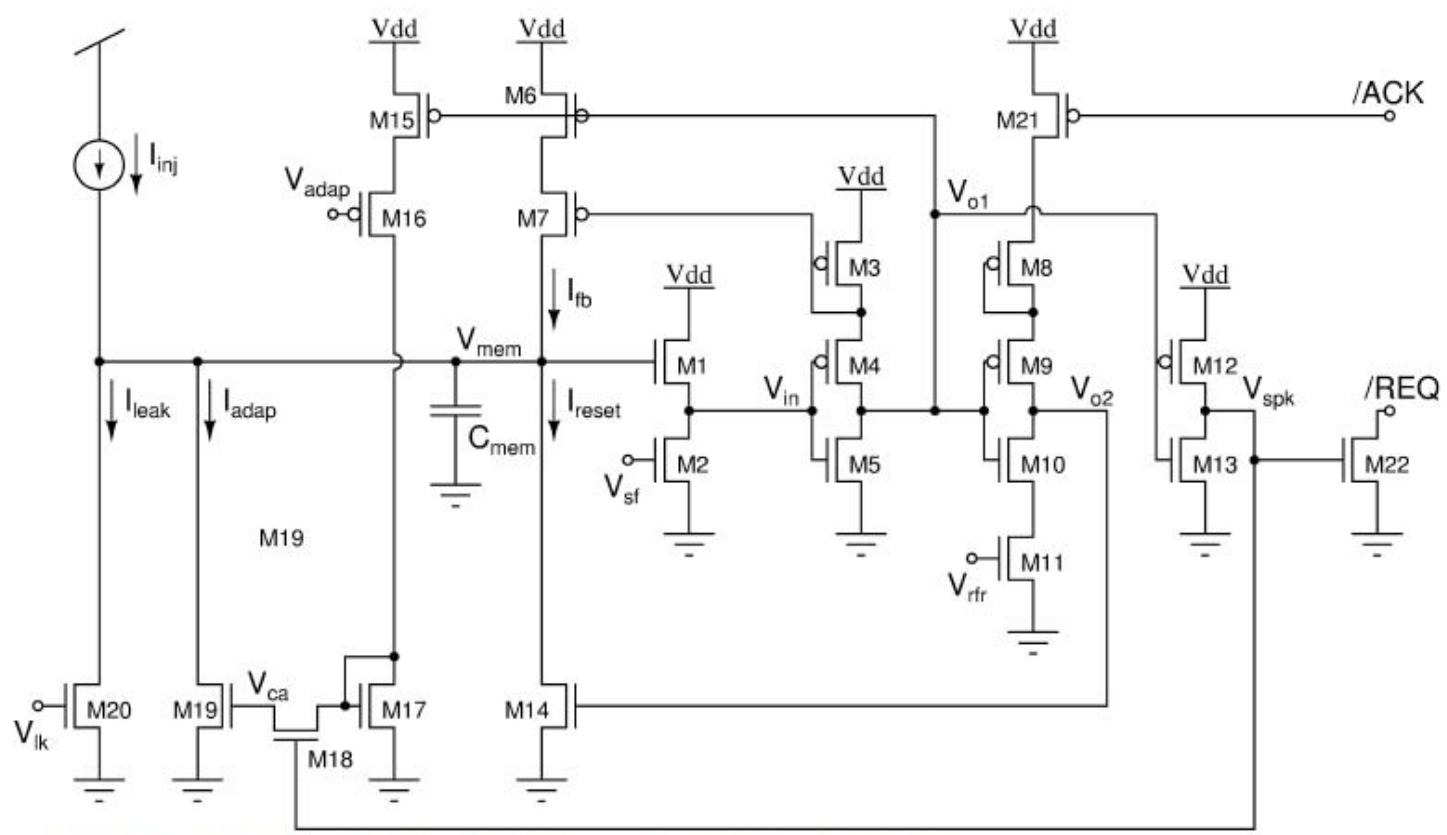

Fig. 2. Circuit diagram of the I\&F neuron. See Section III-A for a detailed description of circuit operation.

Figure 8 Advanced integrate and fire model low power requirements and tunable features [18].

\section{Neuromorphic Model of ACC}

A neuromorphic model of the ACC requires a complete paradigm shift from the neural network models discussed in the previous sections. The primary reason is that the neural network lack physical detail as compared to their intended biological counterparts. Though these models have been widely successful predicting the behavior of the ACC at several levels, including trial-to-trial learning and adaptive 
processing, the physical model of detailed connectivity at the neuron level is lacking. Neuromorphic models inherently demand a more detailed model of the neuron type, synapse connectivity, and process control compared to their neural network cousin. In contrast, a detailed physical model may not be necessary given the success of neural network based approaches, provided the key functional elements are captured in the neuromorphic model. One aspect of this project that makes the feasibility a reality is that the current models of the ACC are control-loop or error driven based. This type of error-driven behavior has been previously demonstrated in neuromorphic models [17, 18].

A control-loop model would be an excellent starting point for a neuromorphic approach. Moreover, key technologies for neuromorphic chip implementations have been developed and are suitable for immediate use. Though these chips are largely based on integrate-and-fire type neurons, they are able to capture a large number of physical effects. Neuromorphic chips (CMOS) are available with imbedded training algorithms, USB connectivity, and software control to configure the problem. The issue of whether it can be implemented is not as critical as to whether the model represents physical brain function. From the previous discussion, though ACC neural network models are clearly not physical, they capture the primary behavior of the ACC conflict control learning mechanism.

\section{Patterning, Topographical, and Organizational Methods for Neural Cell Cultures}

\section{Introduction}

We have focused a portion of this LDRD work on developing a new strategy for organizing living cells into pre-defined arrangements. This technique utilizes buried microfluidic channels in glass substrates that are selectively coated with a molecule that promotes cell attachment. Large cell attachment centers allow cells to attach to the substrate at specific locations, and narrow microfluidic channels connected to these structures permit outgrowth of processes (axons and dendrites) from the cells. The channels also contain pores in the top surface that permit diffusion of medium/reagents to the confined cells. We present evidence that neurons can be effectively guided and directed on these substrates, thus this technique presents a new method for guiding cells using both chemical and topographical cues with an improved capability of longterm guidance given the increased level of confinement. 


\section{Previous Work in the Literature}

(a)

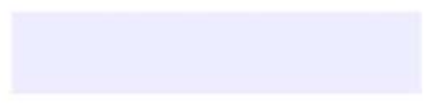

(b)

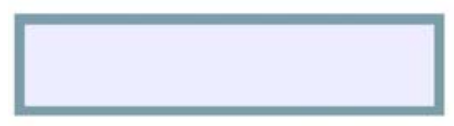

(c)
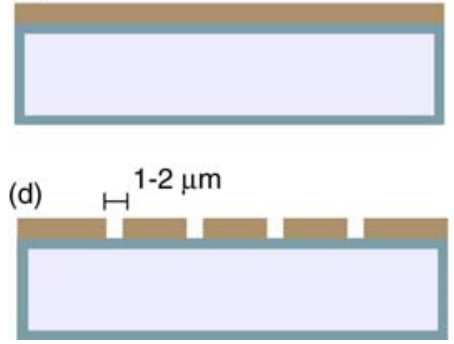

Figure 9 Fabrication process for the buried microfluidic cell guidance cues. (e)

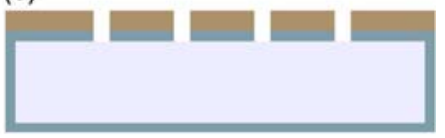

(f)

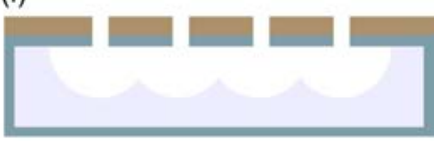

(g)

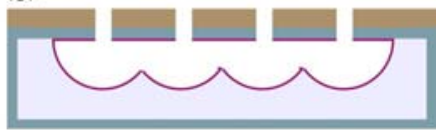

(h)

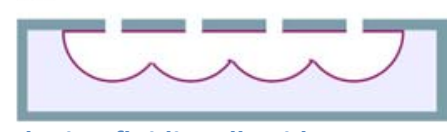

There is an extensive history in the literature for efforts aimed at utilizing microfabricated structures to guide cells, including neurons. Early work by Britland et al. demonstrated the use of topographical and chemical guidance cues for guiding nerve cells [23]. Recent work by ClaverolTinture et al. [24] demonstrated the use of silicone elastomer microfluidic channels for guiding neurons towards recording microelectrodes embedded in the substrate.

Similar approaches have been developed using structures of agarose gel [25], as well as grooves etched into glass substrates [26] and patterned resist [27]. Heuschkel et al. described a method of creating buried microchannels for guiding neurons in pre-defined locations [28].

\section{Device Operation}

The work presented here is unique and novel in its approach, finished structure, and capabilities. Figure 9 shows a schematic of the fabrication process. Initially, fused silica wafers (Figure 9a) are coated in low-stress LPCVD silicon nitride (b). This is a conformal coating that coats the entire wafer, and this material is highly resistant to hydrofluoric (HF) acid which is used to etch the channels later in the processing. The next step is to spin photoresist (c) and then pattern the photoresist with a mask that contains a series of small holes (d). These holes are then transferred into the silicon nitride layer with a $\mathrm{CF}_{4}$ reactive ion etch (e). The next step is to etch the substrate in buffered HF (f), a process which leaves the photoresist and silicon nitride materials intact and removes underlying glass material from the substrate. The spacing of the holes in the silicon nitride layer is crucial to forming buried channels, as the BHF etch is isotropic and closely spaced holes will then merge to form continuous buried microchannels with lids that contain the original holes. The remaining holes will serve as perfusion orifices to allow medium and nutrients to diffuse to growing cells within the buried channels. These pores permit waste to diffuse away from the cells and prevent it from being trapped in close proximity to the cells. After thorough rinsing, the substrate was subjected to glow discharge plasma to increase hydrophilicity to prepare for poly-Llysine deposition. The substrate is immersed in a poly-lysine solution $(1 \mathrm{mg} / \mathrm{ml}$ in borate 
buffer) for 4 hours to overnight (g). The final step is to rinse the substrate in clean buffer and water, and then acetone, isopropanol, and water to remove the photoresist. This leaves poly-lysine only on the surfaces within the channels. Larger holes (15-20 $\mu \mathrm{m}$ in diameter) in the silicon nitride layer are placed adjacent to these buried microchannel features to allow cell bodies to attach and permit axon/dendrite outgrowth along the buried channels. Figure 10 shows a bright-field image of a complete buried guidance cue feature with $15 \mu \mathrm{m}$ wide cell attachment sites. As mentioned previously, these sites are open at the top and allow cells to attach to the bottom of the substrate. This device was BHF etched for 22 minutes to allow pore features to connect and create continuous buried channels. Figure 10b shows an example of a device with 6 pores that were not completely etched all the way through the silicon nitride layer. The pore features are visible (since they are inadvertently partially etched into the nitride) while the undercut regions are not visible as in the nearby pore features. Figure 10c shows an SEM image of a cross-section of a buried channel. The $1 \mu \mathrm{m}$ pore features produce approximately 3.5 $\mu \mathrm{m}$ wide undercut buried channels during a $22 \mathrm{~min}$ BHF etch. The silicon nitride layer is approximately $250 \mathrm{~nm}$ thick and readily withstands the BHF etch. Figure 10d is a crosssection showing the intersection of three buried channels with the cell attachment feature.
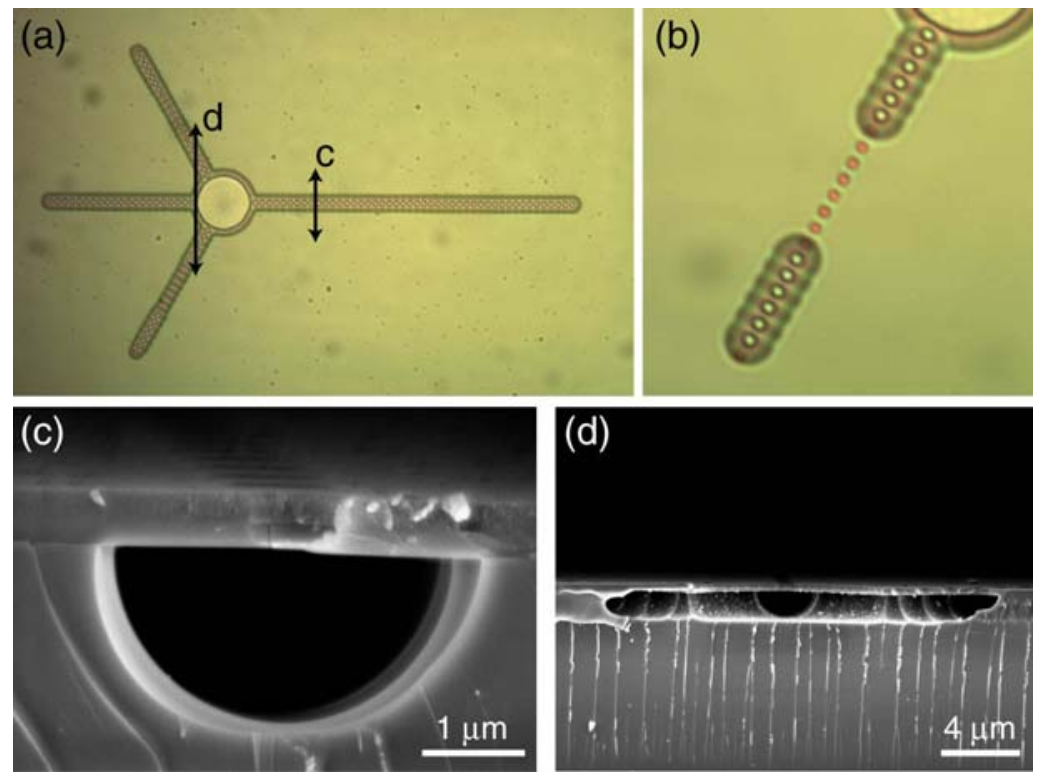

Figure 10 (a) Buried guidance cue features showing the cell body attachment site and neurite outgrowth features. (b) Example of a set of incomplete features. (c) Cross-section of a buried channel at the location c shown in (a).

(d) Cross-section across three channels at the location d shown in (a).

Primary hippocampal neurons have been cultured on these substrates with moderate success. Figure 11 shows examples of cells that have attached and extended processes along the buried guidance cue features. Samples were fixed after six days of culturing. Scanning electron microscopy (SEM) shows evidence that some of the processes are entering the buried channels and are guided along the top surface of the features. Additional guidance cue designs have been developed to promote the connection between multiple neurons guided by interconnected buried features. 


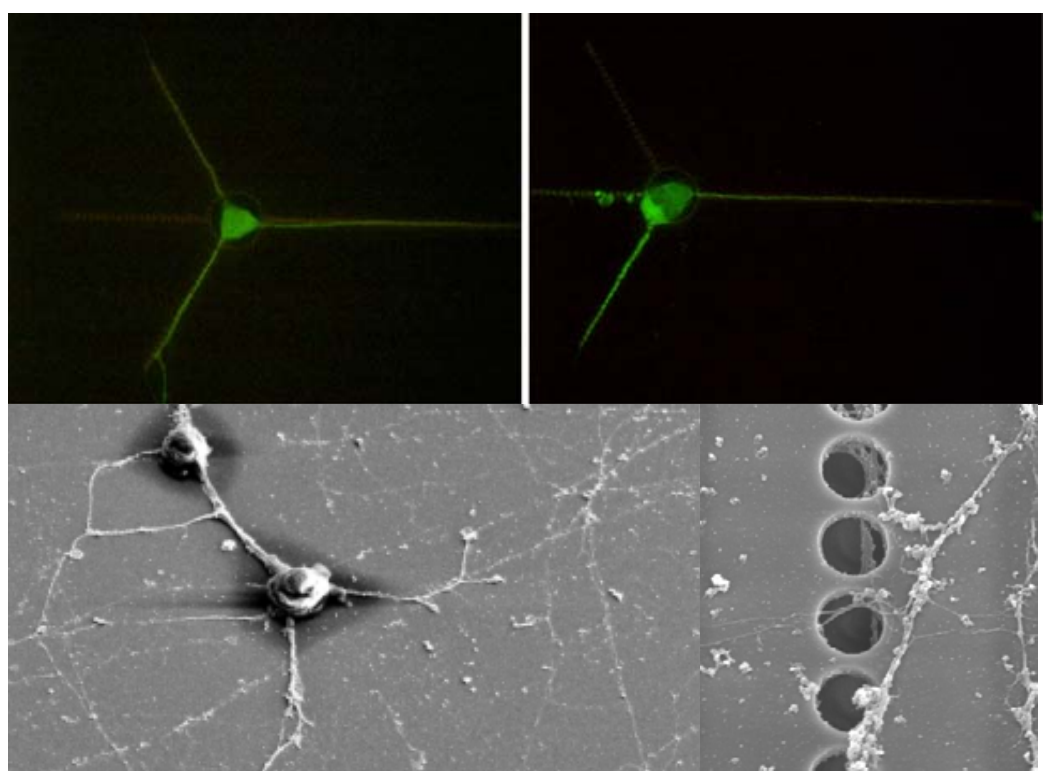

Figure 11 (top) Fluorescence images of neurons grown on the buried channel guidance cue features. (bottom) Scanning electron microscope images on neurons growing on the substrates, showing evidence of cell processes entering the buried channels.

\section{Conclusions}

Based on this review, all the existing models of the ACC were found to be neural network implementations. The major differences in the models were the type of the signals used to represents perceived conflict. The well established models are based conflict-control loop theory of the ACC that suggest trial history impacts level of conflict. More recent models argue that the conflict models fail to account for learned behavior $[7,13]$, whereas the conflict-model proponents argue that there is a lack of experimental data to support error likelihood models. Despite these differences in the ACC models, there is a sufficient understanding of the ACC to model the primary conflict-control behavior. To implement the ACC as a neuromorphic system would require training sets similar to those used in neural networks in the form of congruent, incongruent, neutral, and go/no go type tasks. We expect the primary challenges for success would be translating the neural network equivalents into the neuromorphic chip, either as differing neuron types or altering synapse functionality. A clear benefit of the neuromorphic approach is a more physical representation of the ACC could be created. Clearly, the flexibility or analogous plasticity inherent to neuromorphic chips would be an ideal media to model the behavior of the ACC.

\section{References}


[1] M. M. Botvinick, C. S. Carter, T. S. Braver, D. M. Barch, and J. D. Cohen, "Conflict monitoring and cognitive control," Psychological Review, vol. 108, pp. 624-652, 2001.

[2] C. S. Carter, T. S. Braver, D. M. Barch, M. M. Botvinick, D. Noll, and J. D. Cohen, "Anterior cingulate cortex, error detection, and the online monitoring of performance," Science, vol. 280, pp. 747-749, 1998.

[3] C. S. Carter, A. M. Macdonald, M. Botvinick, L. L. Ross, A. Stenger, D. Noll, and J. D. Cohen, "Parsing executive processes: Strategic versus evaluative functions of the anterior cingulate cortex," PNAS, vol. 97, pp. 1944-1948, 2000.

[4] M. M. Botvinick, L. E. Nystrom, K. Fissel, C. S. Carter, and J. D. Cohen, "Conflict monitoring versus selection-for-action in anterior cingulate cortex," Nature, vol. 402, pp. 179-181, 1999.

[5] B. J. Casey, R. J. Orendi, J. L. Schubert, A. B. Nystrom, L.E.Nystrom, J. N. Castelanos, F. X. Haxby, J. V. Noll, D. C. Cohen, S. D. Forman, R. E. Dahl, and J. L. Rapoport, "A developmental function MRI study of prefrontal activation during performance of a go-no-go task," J. Cognitive Neuroscience, vol. 9, pp. 835-847, 1997.

[6] S. Ito, V. Stuphorn, J. W. Brown, and J. D. Schall, "Performance monitoring by the anterior cingulate cortex during saccade countermanding," Science, vol. 302, pp. 120-122, 2003.

[7] J. W. Brown and T. S. Braver, "Learned predictions of error likelihood in the anterior cingulate cortex," Science, vol. 307, pp. 1118-1121, 2005.

[8] U. Mayr, E. Awh, and P. Laurey, "Conflict adaptation effects in the absence of executive control," Nature Neuroscience, vol. 6, pp. 450-452, 2003.

[9] C. S. Carter and V. v. Veen, "Anterior cingulate cortex and conflict detection: An update of theory and data," Cognitive, Affective, and Behavioral Neuroscience, vol. 7, pp. 367-379, 2007.

[10] A. D. Jones, R. Y. Cho, L. E. Nystrom, J. D. Cohen, and T. S. Braver, "A computational model of anterior cingulate function in speeded response tasks: Effects of frequency, sequence, and conflict," Cognitive, Affective, and Behavioral Neuroscience, vol. 2, pp. 300-317, 2002.

[11] T. S. Braver, D. M. Barch, J. R. Gray, D. L. Molfese, and A. Snyder, "Anterior cingulate cortex and response conflict: Effects of frequency, inhibition, and errors," Cerebral Cortex, vol. 11, pp. 825-836, 2001.

[12] N. D. Pisapia and T. S. Braver, "A model of dual control mechanisms through anterior cingulate and prefrontal cortex interactions," Neurocomputing, vol. 69, pp. 1322-1326, 2006.

[13] J. W. Brown and T. S. Braver, "Risk prediction and aversion by anterior cingulate cortex," Cognitive, Affective, and Behavioral Neuroscience, vol. 7, pp. 266-277, 2007.

[14] A. Simmons, S. C. Matthews, J. S. Feinstein, C. Hitchcock, M. P. Paulus, and M. B. Stein, "Anxiety vulnerability is associated with altered anterior cingulate response to an affective appraisal task," Brain Imaging, vol. 19, pp. 1033-1037, 2008. 
[15] K. A. Boahen, P. P. Pouliquen, A. G. Andreou, and R. E. Jenkins, "A heteroassociative memory using current-mode MOS analog VLSI circuits," IEEE Trans. Cir. and Sys., vol. 36, pp. 747-755, 1989.

[16] K. A. Boahen and A. G. Andreou, "Design of a bidirectional associative memory chip," in Associative neural memories: Theory and implementation, M. Hassoun, Ed. New York: Oxford univ. Press, 1993, pp. 288-305.

[17] R. Sarpeshkar, C. Salthouse, J.-J. sit, M. W. Baker, S. M. Zhak, T. K.-T. Lu, L. Turicchia, and S. Balster, "An ultra-low-power programmable analog bionic ear processor," IEEE Trans. Biomed. Eng., vol. 52, pp. 711-727, 2005.

[18] G. Indiveri, E. Chicca, and R. Douglas, "A VLSI array of low-power spiking neurons and bistable synapses with spike-timing dependent plasticity," IEEE Trans. Neural Networks, vol. 17, pp. 211-221, 2006.

[19] S.-C. Liu, "A silicon retina with controllable winner-take-all properties," in IEEE Int. Symp Circuits and Systems. Bangkok, Thailand, 2003.

[20] S. Fusi, M. Annunziato, D. Badoni, A. Salamon, and D. Amit, "Spike-driven synaptic plasticity: Theory, simulation, VLSI implementation," Neural Computing, vol. 12, pp. 2227-2258, 2000.

[21] H. Markram, J. Lübke, M. Frotscher, and B. Sakmann, "Regulation of synaptic efficacy by coincidence of postsynaptic AP's and EPSPs," Science, vol. 275, pp. 213-215, 1997.

[22] L. F. Abbott and S. Song, "Asymmetric Hebbian learning, spike timing and neural response variability," Adv. Neural Inf. Process. Syst., vol. 11, pp. 69-75, 1999.

[23] S. Britland, C. Perridge, M. Denyer, H. Morgan, A. Curtis, and C. Wilkinson, "Morphogenetic guidance cues can interact synergistically and hierarchically in steering nerve cell growth," Experimental Biology Online, vol. 1, pp. 1-15, 1997.

[24] E. Claverol-Tinture, M. Ghirardi, F. Fiumara, X. Rosell, and J. Cabestany, "Multielectrode arrays with elastomeric microstructured overlays for extracellular recordings from patterned neurons," J. Neural Eng, vol. 2, pp. 1-7, 2005.

[25] I. Suzuki, Y. Sugio, H. Moriguchi, Y. Jimbo, and K. Yasuda, "Modification of a neuronal network direction using stepwise photo-thermal etching of an agarose architecture," Journal of Nanobiotechnology, vol. 2, pp. 7, 2004.

[26] J. W. Lee, K. S. Lee, N. Cho, B. K. Ju, K. B. Lee, and S. H. Lee, "Topographical guidance of mouse neuronal cell on $\mathrm{SiO}_{2}$ microtracks," Sensors \& Actuators: $B$. Chemical, vol. 128, pp. 252-257, 2007.

[27] H. Francisco, B. B. Yellen, D. S. Halverson, G. Friedman, and G. Gallo, "Regulation of axon guidance and extension by three-dimensional constraints," Biomaterials, vol. 28, pp. 3398-3407, 2007.

[28] M. O. Heuschkel, L. Guerin, B. Buisson, D. Bertrand, and P. Renaud, "Buried microchannels in photopolymer for delivering of solutions to neurons in a network," Sensors \& Actuators: B. Chemical, vol. 48, pp. 356-361, 1998. 


\section{Distribution List}

$\begin{array}{ll}1 & \text { MS } 1425 \\ 1 & \text { MS } 1188 \\ 1 & \text { MS } 1425 \\ 1 & \text { MS } 1425 \\ 1 & \text { MS } 0899\end{array}$

Darren W. Branch

Chris Forsythe

Conrad D. James

Steve Casalnuovo

Technical Library (electronic), 9536 